\title{
HEP in Greek Classes
}

\section{Dimitris Fassouliotis ${ }^{1}$, Christine Kourkoumelis ${ }^{1}$, Stylianos Vourakis ${ }^{1}$}

1 Physics Faculty and Institute of Accelerating Systems and Applications (IASA), National and Kapodistrian University of Athens (NKUA), Panepistimioupoli, Ilissia 15771, Greece

\begin{abstract}
The HEP Inquiry learning resources created over the last four years by the European outreach projects are reviewed. The resources are mostly addressed to high school students and the purpose is to ignite their interest on science. In addition, at the University of Athens for the last four years we have been using the HYPATIA on-line event analysis tool as a lab course for fourth year undergraduate physics students, majoring in HEP. Each year 40-50 students highly appreciated the course, since they get a direct involvement in the actual top-level research. Up to now, the course was limited to visual inspection of a few tens of ATLAS events. Recently we have enriched the course with additional analysis exercises, which involve large samples of events. The students, through a user friendly interface can analyze the samples (both signal and background ones) and optimize the cut selection in order to search for the Higgs decay $\mathrm{H}_{\square} 4$ leptons. Recently ATLAS released $1 / \mathrm{fb}$ of data, so starting now the students analyse real data.
\end{abstract}

\section{Introduction}

We, as HEP researchers and university teachers, feel that it is our duty to get the general public, the students and the teachers to get to know better what Big Science is about. The recent discovery (2012) of the Higgs boson has attracted large media coverage generating great public interest. We should maintain and feed this interest and momentum by continuously provoking students' and public's interest in HEP. There has been in general a decreasing interest of young people towards science education subjects, which is decreasing with age as well. How can we attract their interest back to STEM or STEAM subjects? As far as HEP is concerned, the challenge is even harder since in most countries HEP is absent from the national school curricula.

In Greece and worldwide, a wide program of different activities has been designed and worked out during the last ten years or so towards this goal.

To that end, activities which tend to ignite the students' interest on science, which involve masterclasses, science fairs and science exhibitions as well as resources for the teachers to use in their class will be briefly reviewed in this contribution.

\section{CERN exhibitions}

During the last five years, CERN exhibitions were hosted twice in Greece. The revised CERN mini-exposition toured Greece during 2011-2012 visiting six cities (Athens, Heraklion, Patras, 
Alexandroupolis, Kavala and Volos) in all parts of the country. In eight months there have been about 22,000 student visitors who were introduced to CERN and its research accompanied in most cases by their teachers as well as a large crowd of general public especially in smaller cities where there are few educational/cultural events.

About two years later, spring 2014, the "Accelerating Science" CERN exposition came to Athens for a month and a half. It was hosted at the premises of the Eugenides Foundation [1], science museum and planetarium). The target group of this large exhibition was: school students 9-17, teachers, university students, the general public and the families interested in engaging their children with science. We trained and provided guides during the 50 day duration of the exhibition. It was a huge success with more than 11,000 visitors, among them 4,000 students from 92 schools; some of which came from as far as $500 \mathrm{~km}$ away. The exhibition was inaugurated in a well-attended ceremony (Figure 1) just two days after the visit of the (then) President of the Greek Republic Mr. K. Papoulias at CERN. The visit was the first of its kind in the 60 year existence of CERN.

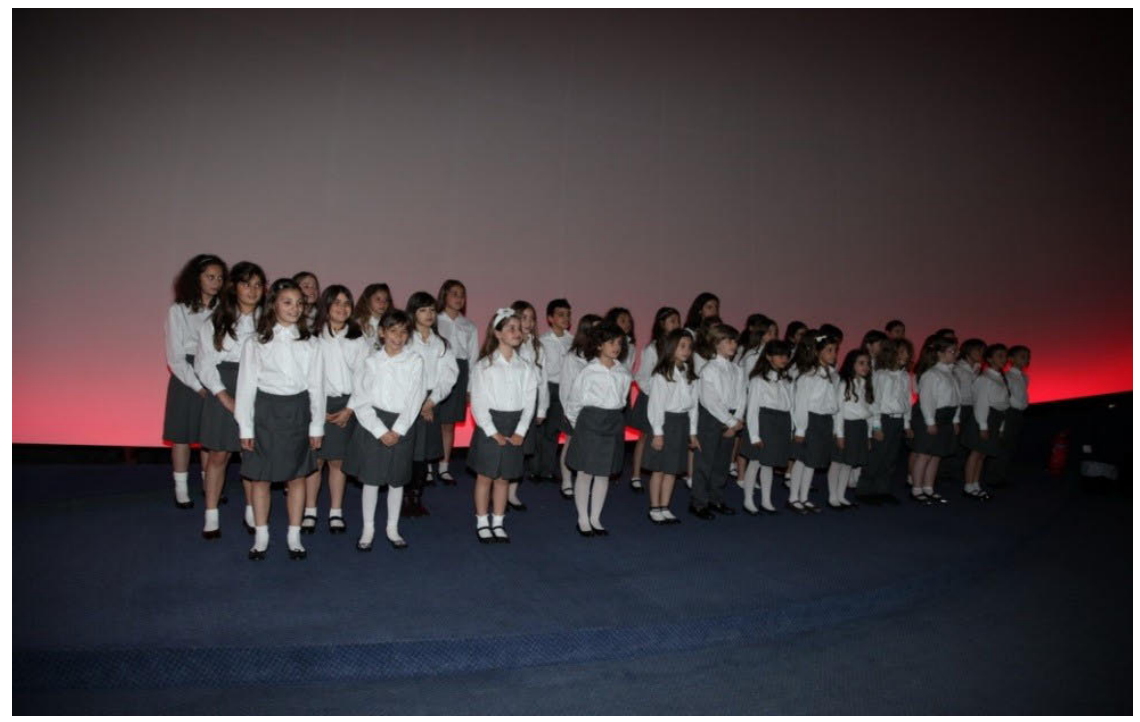

Figure 1. The chorus of the Ellinogermaniki school [2] singing the "Higgs boson" song composed by the elementary school children during the inauguration event of the "Accelerating Science" exposition.

For both exhibitions a great effort was dedicated in order to enhance the educational value of the exhibitions by training teachers in local workshops and introducing them to resources which they could carry back to their school in order to involve their students in activities which would simulate the work of the researchers at CERN. During the mini-exposition we trained about 750 teachers in local dedicated seminars and during the "Accelerating Science" we trained about 120 teachers in hands-on workshops at the Eugenides foundation.

\section{Science fairs in Greece}

In the context of Intentional Conferences taking place at various parts of Greece, we have been organizing local science fairs which are mainly addressed to younger students and their families.

In parallel with the ICNFP (International Conference for New Frontiers in Physics) which takes place every summer at Kolymbari, close to the city of Chania in Crete, we organized for four consecutive years a science fair at the premises of a fantastic setting, the Chania Sailing Club at the waterfront of the old port. During the CONF12 and under its auspices we organized two science fairs: 
one in the central public library of the city of Veria (about $50 \mathrm{~km}$ west of Thessaloniki) and a second one at the New Town Hall of Thessaloniki. The Veria public library is very popular to its citizens since it constantly holds events and was awarded the 2010 Bill Gates Foundation prize. The format of the above fairs/events was the following: simple hands-on experiments designed by Quarknet [3] for young kids (conservation of momentum, rolling with Rutherford, probe matter, quark workbench, W signatures), plus several puzzles, books, card games etc. In addition there was a demonstration of PC based Masterclasses (ATLAS and ALICE) for high-school kids. Later in the day the fairs were concluded by an evening public lecture (in Greek) on hot topics (this year was about the gravitational waves) and a music performance. All fairs were very well attended despite the fact that they were held in July/August when the schools were closed and families were on vacation.

\section{European and International efforts/resources}

The European Union (EU) is supporting outreach projects which could increase young students' interest in science by providing a connection between formal training with the informal learning targeted towards active research. An effective way to stimulate students' interest in STEM subjects is to actively involve them in hands-on top level research and discoveries.

The authors have been very actively engaged in several EU outreach programs over the last ten years which created thousands of educational scenaria. Some have already been finished but the resources are ready to be used at the corresponding portals. Below we briefly mention the projects with references to their resources:

1) Learning with ATLAS@CERN (2009-2011). The project was coordinated by one of the authors; its main objective was to use the information material and the data from the ATLAS experiment in order to build educational scenarios for schools and universities. It formed a rich repository of HEP resources relevant to the ATLAS experiment [4]. This way it demonstrated and applied the innovative pedagogical approach of inquiry learning to HEP oriented scenaria for students, teachers and the general public.

2) PATHWAY to inquiry based science education (2011-2013). The project focused on teachers and proposed a standard-based approach to teaching science, in general and not only HEP, by inquiry. It outlined instructional models, motivated the teachers to adopt inquiry based techniques and activities in their classrooms, and offered access to a unique collection of open educational resources and teaching practices [5].

3) Discover the COSMOS: e-Infrastructures for an Engaging Science Classroom (2010-2012) which was coordinated by one of the authors. The Discover the COSMOS activities introduced students to concepts and ideas of big science in the fields of Astronomy and HEP. The project developed an innovative learning environment [6] which brought together more than 95,000 science education learning objects and activities connected to the science curriculum from Astronomy, Space Physics and HEP, and was later integrated to the Inspiring Education Portal.

4) Go-lab [7]: The project finished at the end of October 2016 after four years. Its goal was to open up the remote science laboratories, their data archives, and virtual models ("online labs") for largescale use in education. Go-lab was addressed to students, teachers and lab-owners. During its operation it created a federation of 385 on-line labs, 343 Inquiry Learning Spaces and 37 Apps in all STEM curricula subjects in 10 languages. Four HYPATIA scenarios (see below) are included in the Go-lab portal as Inquiry Learning Spaces (ILSs).

5) Inspiring Science Education (ISE). The ISE project [8] began in April 2013 and ended last July. ISE designed, planed and implemented large-scale pilots to stimulate and evaluate innovative use of existing eLearning tools and digital resources for scientific disciplines and technology (STEM related subjects), enhancing science learning in 5,000 primary and secondary schools in 15 European countries. Currently there are 186 demonstrators (full educational scenaria). In addition to the demonstrators, ISE has harvested existing repositories with 278,000 educational resources (mainly Open Discovery Space [9] and Discover the COSMOS portal [10]). 
6) Creations [11] is the only ongoing project presently. It started about a year ago and will continue for two more years. It aims to develop creative approaches based on art in order to increase young people's interest in science. The project will use the combination of art and science in order to enhance a cross-disciplinary thinking and raise student's interest in both. The sixteen partners from ten European countries are planning a variety of events with theatre, photography, exhibitions in which young people can experience an active and playful role within science and research.

\section{HEP Masterclasses}

In spring 2016, the 12th edition of IPPOG's [12] International Masterclasses (IMC) [13] took place. They are addressed to high school students aged between 15 and 19 and the main idea behind them is to give them the opportunity to perform top-level research, namely to become "scientists for one day". The format is the following: every year the selected students are invited to a research institute or university, listen to introductory talks followed by a two hour long measurement using LHC data from any of the four experiments (ATLAS, CMS, ALICE, LHCb). The day ends with an international video conference where students present at two to five institutes show, compare and discuss their results with the help of two moderators centrally placed either at CERN or Fermilab. Last spring 46 countries, 213 institutes, 13,000 high-school students and 1,100 teachers took part; a really worldwide effort. Although as mentioned above the LHC data analysis involved all four experiments, the ATLAS analysis offered two different exercises the so called analysis "paths", the Z-path [14] and the W-path [15]. The Z-path which uses the HYPATIA tool [16], written by the NKUA/IASA group and described below, is by far the most popular since $30 \%$ of all IMC's of all four experiments, use it. In Greece the masterclasses took place in three different large cities: Athens, Thessaloniki and Heraklio (Crete). In Athens three institutes participated: NKUA (60 students), N.R.C. Democritos (40 students) and NTUA (120 students plus 30 teachers). It is worth mentioning that the applications far exceeded the number of available places; in Athens there were applications from 74 schools $(\sim 370$ students) for 220 places. In both Thessaloniki (AUTH) and Crete (UoC) there was also a high number of applications and 100 students participated in each premise.

Recently more masterclasses using mainly non-accelerator data such as cosmic rays and neutrinos have been designed and are taking place for first time. The South Pole Icecube neutrino experiment held a masterclass [17] last spring. The accompanying Icetop cosmic ray airshower detector provides an online masterclass [18] with mixed data and simulated events. DESY has been coordinating the International Cosmic day [19] since 2012. The format is similar to the IMCs but the students analyse data from different detectors in order to measure cosmic particles, in particular the zenith angle distribution of the air showers. They try to answer questions such as: what are the cosmic particles? where do they come from? how can they be measured? The Quarknet among its numerous outreach activities organizes since 2015 an International Muon Week each year [20]. The participants share data worldwide, measure the cosmic ray flux all over the world and compare their measurements at the end of the week. Quarknet is also preparing a masterclass to analyse data from the neutrino detectors at Fermilab (MINERVA and Nova). Furthermore, the Pierre Auger observatory which is searching for giant air showers has publicly released $1 \%$ of their data to be used in masterclasses [21]. Finally there is also a very recent masterclass being prepared based on gravitational wave searches from the European Gravitation Observatory.

To complete the above list, although is not a masterclasses, the CERN's Beamline for schools competition [22] is a very important outreach event with hundreds of participating teams each year from many countries across the world. The competition started in 2014 as part CERN's 60th anniversary celebrations. The competing teams of high school students are normally guided by a teacher and design an experiment which uses a fully equipped beam line (including various detectors) at CERN's Proton Synchrotron. Up to now more than 5,500 school students have participated. In the first edition of the competition a Greek team from the Varvakios Innovative School of Athens was one of the two winning teams, out of about 300 participants! 


\section{National Masterclasses}

Besides the IMCs which take place once a year, we have been conducting national and local masterclasses in a great number of schools across the country. Thanks to the support of the above mentioned EU outreach programs, we have been able to promote the use of existing ICT, new methodologies and new eLearning tools ready to be used in the classroom, train the teachers in intergrading inquiry learning science education in the classrooms and this way gradually change their teaching approach. We have also built teacher communities so that teachers can interact and exchange their experiences. The material we developed is ready to be used by the trained teacher in the duration of a school lesson and engages the learner in scientifically oriented questions.

We have determined [23-26] that the best practice for the national masterclasses which introduce HEP to 16-18 year old students is a combination of lectures, virtual tours to specific experiments and hands-on experience with the HYPATIA tool, which is described below. This combination has taken the form of the so called "mini masterclasses" which are half-day workshops, taking place locally at the interested schools. During the last 4-5 years we have run "mini masterclasses" in about 200 schools across the Greece. We have also built resources based on the games of CERNLand for younger kids aged 9-12 and 12-15. Figure 2 is a map of the locations of national masterclasses which took place during the 2014-2016 school years. The size of each dot is proportional to the number of students who participated.

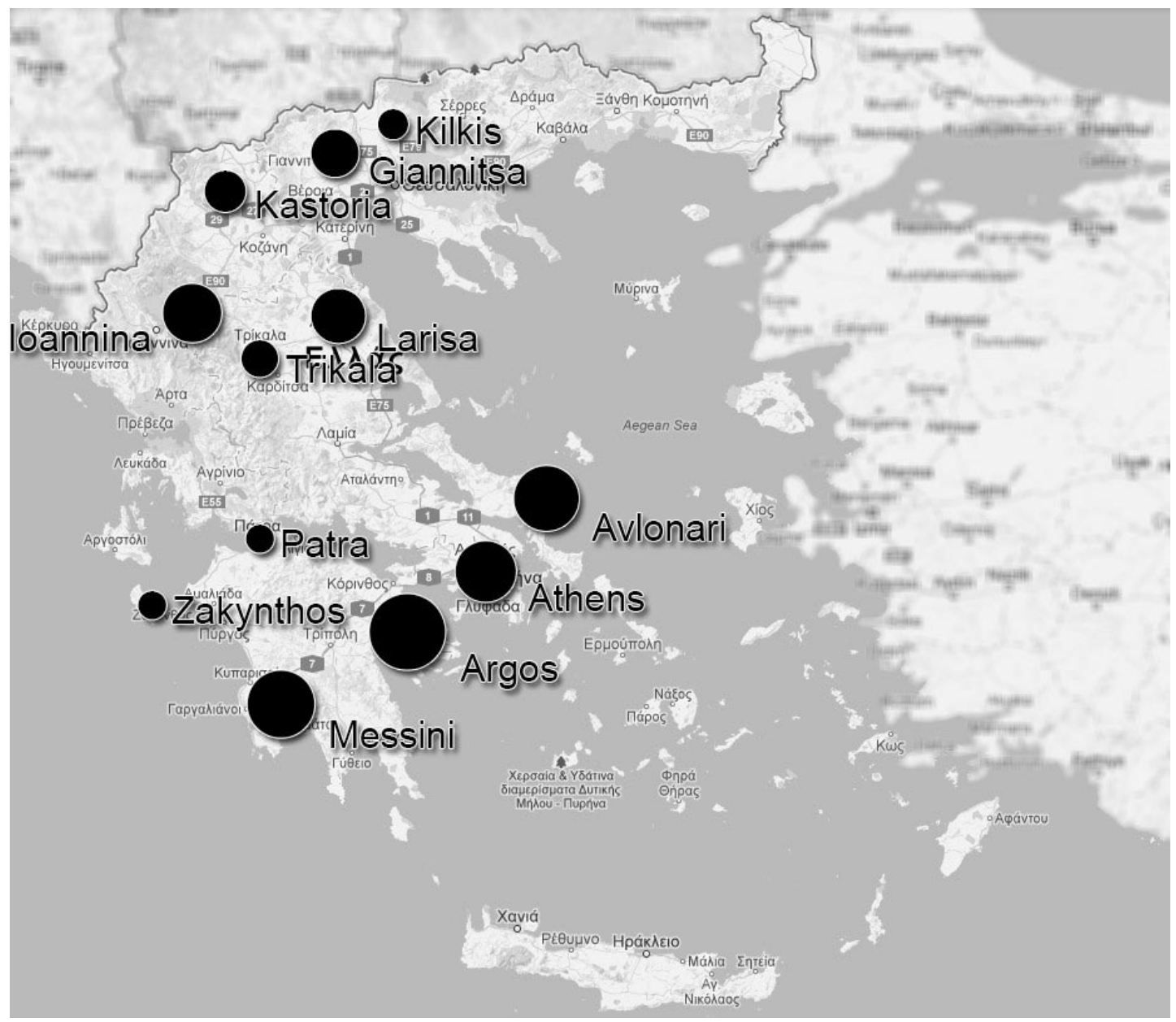

Figure 2. The size of each dot is proportional to the number of answers. The implementation took place in different school environments in urban, sub-urban and rural areas of Greece. 
For interested remote schools which either was not practical to visit or did not have the time for a full mini-masterclasses we interconnected them with other schools which we were actually visiting through a virtual visit to either the ATLAS [27] or CMS [28] experiment.

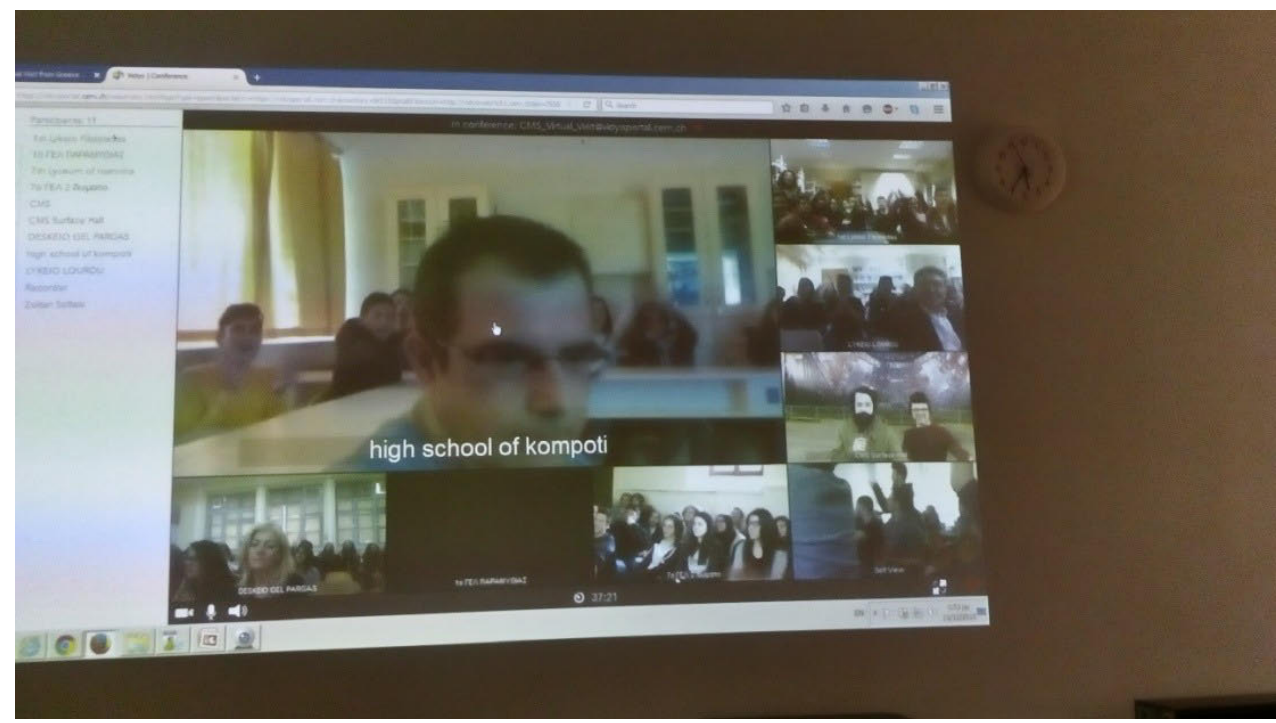

Figure 3. Six schools from the Irirus region of Greece connected for a virtual visit to the CMS experiment.

Figure 3 is a screenshot of six schools connected simultaneously to the CMS control room, all of them from the northwest part of Greece. Members of the group were present and conducted masterclasses in three schools and the rest were connected only through videoconferencing and followed the event. Here it is worth mentioning that last year the Greek schools had the highest number of virtual visits to the LHC experiments than any other country.

\section{The HYPATIA tool}

HYPATIA (HYbrid Pupil's Analysis Tool for Interactions in ATLAS) was first written more than ten years ago by the NKUA/IASA outreach group. HYPATIA is a graphical representation of the products of proton collisions registered by the ATLAS detector. Students using the event display can interact with the events and in this way study the fundamental building blocks of nature and their interactions and try to make discoveries using a playful tool for learning.

The first version of HYPATIA was the full-featured offline version based on the ATLANTIS event display [29]. The full version of HYPATIA [30] has been used in the IMCs Z-path since 2009 worldwide. It uses java and is therefore compatible with most operating systems (Windows, Linux, OSX, Solaris, etc.). This version is updated every year with new functionality to better serve the IMCs needs as the Z-path exercise evolves.

In addition to the offline version, since 2010 we have developed an online version of HYPATIA [31] which is simpler, does not need to be downloaded and is aimed at educational use in schools and universities. Since 2014 the version runs as a web application in any browser including mobile devices. A few months ago it was awarded the GOLC award (Figure 3) for the "best visualized experiment" 


\section{Global Online Laboratory Consortium}

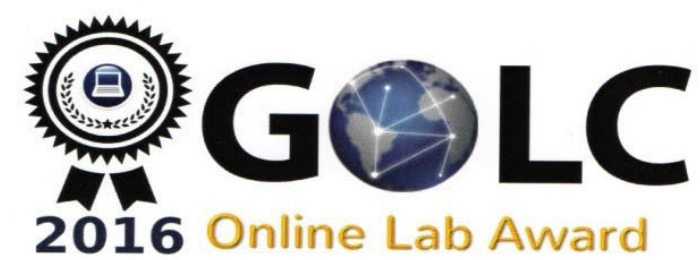

The GOLC Online Laboratory Award 2016 in the category

"Visualized Experiment"

is presented to

HY.P.A.T.I.A. Event Display

Submitted by:

Christina Kourkoumeli and Stylianos Vourakis

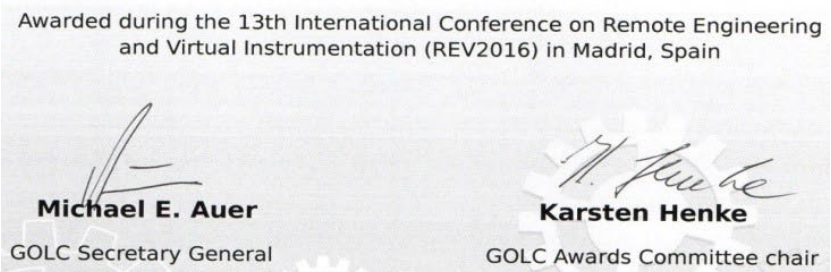

Figure 4. The 2016 GOLC award for the HYPATIA online tool

We have been creating different personalized exercises/labs involving HYPATIA. Some of them are directly connected to the school curricula. As an example, in the Go-lab portal there is scenario which uses the giant ATLAS solenoid magnet and the curvature of the particles in the central detectors to teach students about Lorentz force. Another scenario uses the fact that on the plane perpendicular to the colliding beams, the momentum before the proton collisions is zero, therefore it should be zero after the collisions as well. The students add the momenta of the produced particles and use the conservation of momentum to draw conclusions about the possible production of neutrinos. The most commonly used scenario involves the hunt for $\mathrm{Z}$ and Higgs bosons.

In line with the requirements of the ISE EU project we have also designed a "special" HYPATIA scenario the so called demonstrator. Each demonstrator is a complete lesson plan structured in several phases of the inquiry learning: Orienting and Asking Questions, Hypothesis Generation and Design, Planning and Investigation, Analysis and Interpretation and Conclusion and Evaluation. In order to evaluate the benefits and effectiveness of each phase we have also designed a number of assessment questions which accompany each phase and which are adopted according to the PISA 2012 Problem Solving Competence Framework. During the last one and a half year we have run the HYPATIA ISE demonstrator in about 76 Greek schools which participated in the implementation (of about two-hour duration) in the framework of their normal curriculum. The data collected from about 270 students' answers demonstrate that the students involved in these activities show high proficiency level in their problem solving competences. In all cases students are scoring also much higher than the OECD average scores which are used as a reference. 


\section{The HYPATIA tool for University students}

Most of the available outreach tools mentioned above are addressed to high school students or even younger, kids. Up to the present day, very few universities offer HEP lab courses. We at the NKUA have decided to expose our undergraduate students to state-of-the-art research by building tools targeted towards them in a three hour long laboratory exercise. The first exercise (out of the two) had the students acquainted with the event browsing and visual inspection of events, using the online HYPATIA tool. They performed particle type identification tasks, invariant mass calculations of unstable particles out of their decay products and used statistical tools in order to claim a discovery. The second part - which was completed very recently-simulates in a pragmatic way the analysis done by CERN researchers in order to separate signal from noise and eventually make a discovery [32].

The ATLAS experiment has released $1 \mathrm{fb}^{-1}$ of their Run I data for educational purposes. The data can be found in the ATLAS open data page [33]. Using the HYPATIA online tool we have created a "batch mode" option, where the user can process very fast a large number of events. The exercise consists of optimizing the criteria -out of a provided list- for maximizing the signal to background ratio. The optimization is done with the so called " $n-1$ " method, by examining distributions of kinematical variables of samples of simulated signal and background events and maximizing the significance which is equal to $\sqrt{2 *\left((S+B) * \ln \left(1+\frac{S}{B}\right)-S\right)}$.

After the selection of the final optimized cuts, the distribution of simulated data plus background is compared the real ATLAS data. The studies which we have implemented are the $Z$ boson decays to two leptons and the Higgs decay to four leptons. Figure 5 shows the optimization of two criteria (the pT of the second lepton and the isolation of the less isolated lepton) for the $\mathrm{Z}$ simulated samples. Figure 6 shows separately the reconstructed invariant mass of the two lepton samples for simulated signal (blue) and simulated background (green) before and after the optimization of cuts. Full documentation is provided for the lab, including a ten-page instruction manual [34] and two videos [35]. The lab is also now linked from the new ATLAS open data resource pages [35]. The "batch mode" has been running with great success since last year for our third year students who major in particle physics. This year the comparison to real data and the two lepton analysis has been added, since after the criteria optimization the four lepton real data mass has only a handful of events to compare with.

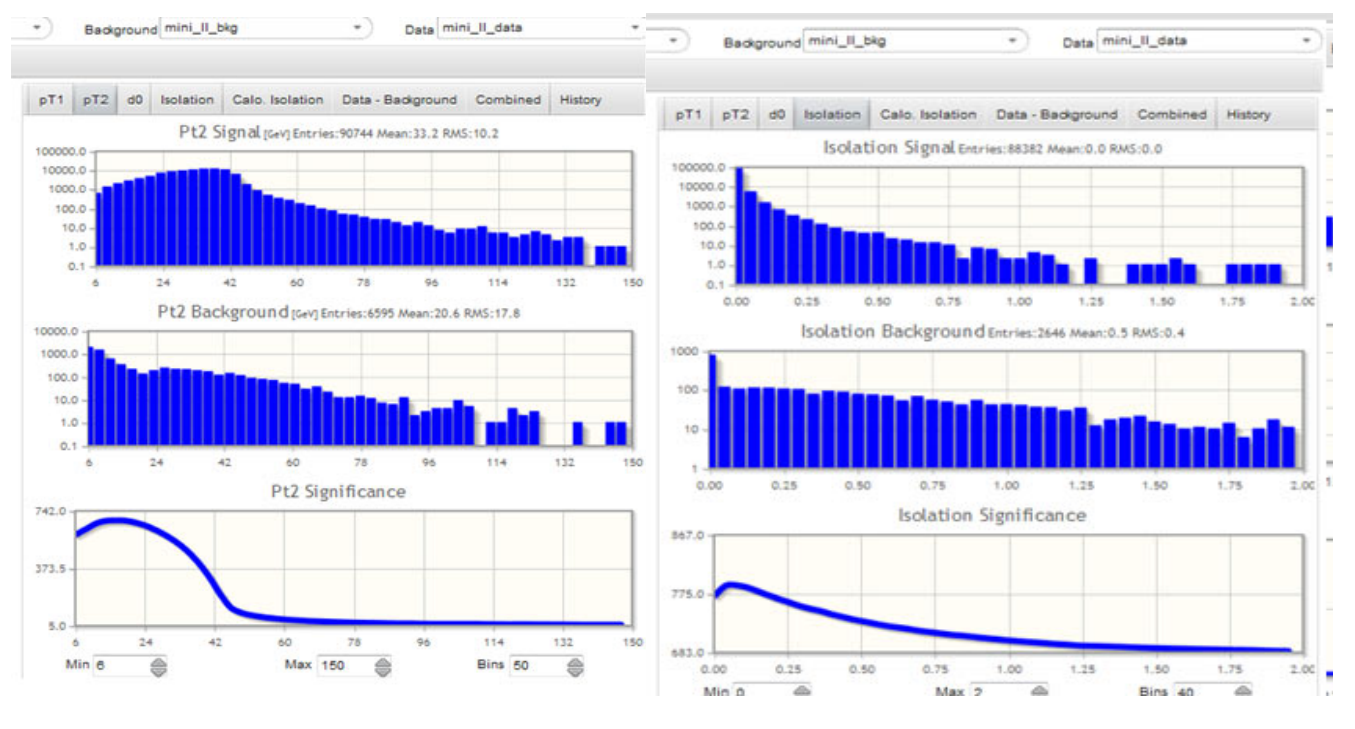


Figure 5. The above plots show the optimization of the $\mathrm{p}_{\mathrm{T} 2}(\mathrm{left})$ and the isolation (right) criteria. The top plots are the distributions from simulated signal $\mathrm{Z}$ bosons, middle plots are the respective distribution for the simulated background events and the one below is the distribution of the significance variable.

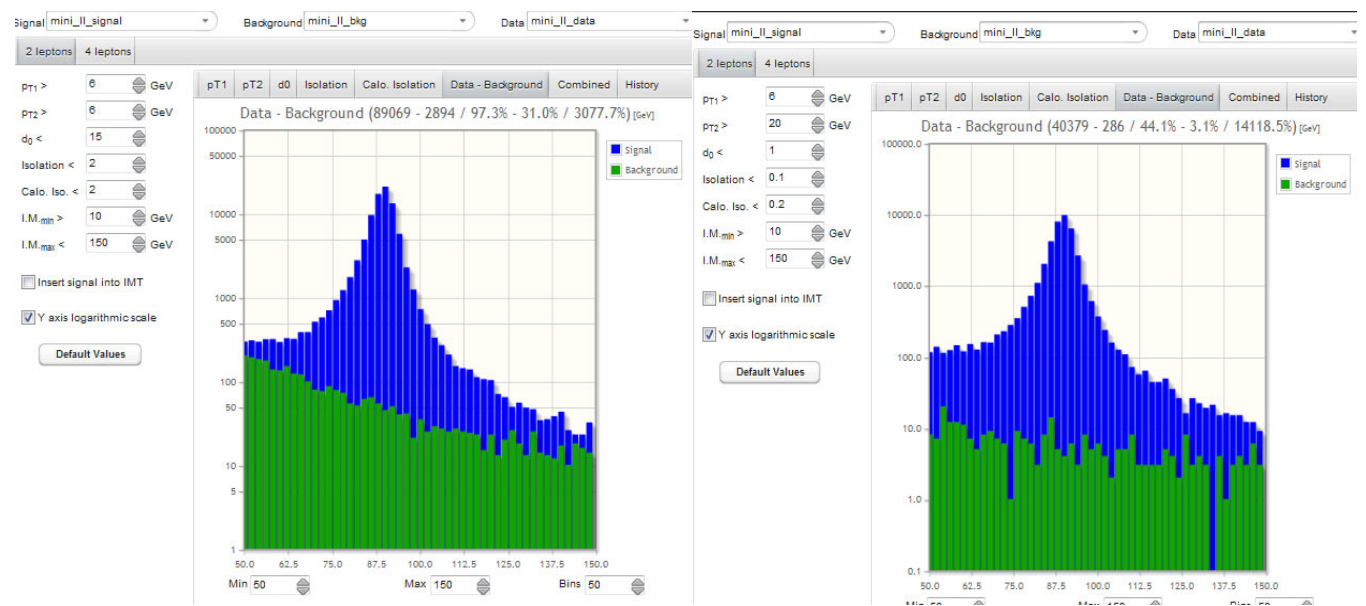

Figure 6. The invariant mass of the two leptons from simulated samples of $Z$ boson signal (blue) and background (green). The left plot is the one with default criteria and the right plot is the one with optimized criteria.

\section{Conclusions}

There is a plethora of outreach activities in Greece in the context of EU programs, the IPPOG IMCs and the international HEP conferences organized in Greece. Thanks to the local "mini-masterclasses" students from urban, sub-urban and rural areas of Greece get to know about HEP research by interactively using hands-on analysis tools. More advanced HEP analysis techniques have also been designed for use at the University level.

\section{Acknowledgements}

This work was partially funded by the European Union in the context of the CREATIONS project (Grant Agreement no. 665917) under the Horizon 2010 program. This document does not represent the opinion of the European Union, and the European Union is not responsible for any use that might be made of its content. The authors would also like to thank Dr.S.Sotiriou of Ellinogermaniki Agogi School and his R\&D group for help and support during all the National Masterclasses.

\section{References}

1. [Online] http://www.eugenfound.edu.gr

2. [Online] www.ea.gr

3. [Online] https://quarknet.i2u2.org/home

4. [Online] http://www.learningwithATLAS-portal.eu.

5. [Online] http://www.pathway-project.eu/

6. [Online] http://www.discoverthecosmos.eu/

7. [Online] http://www.go-lab-project.eu/

8. [Online] http://inspiringscience.eu/

9. [Online] http://opendiscoveryspace.eu/

10. [Online] http://www.portal.discoverthecosmos.eu

11. [Online] http://creations-project.eu/ 
12. [Online] http://ippog.web.cern.ch/

13. [Online] http://physicsmasterclasses.org/

14. [Online] http://atlas.physicsmasterclasses.org/en/zpath.htm

15. [Online] http://atlas.physicsmasterclasses.org/en/wpath.htm

16. Kourkoumelis, C., \& Vourakis, S. (2014). Hypatia — an online tool for ATLAS event visualization. Physics Education, 49, 21-32

17. [Online] http://icecube.wisc.edu/masterclass/home

18. [Online] http://icecube.wisc.edu/viewer/icetop

19. [Online] http://icd.desy.de

20. [Online] https://quarknet.i2u2.org/group/international-muon-week

21. [Online] http://auger.colostate.edu/ED/

22. [Online] cern.ch/bl4s

23. C.Kourkoumelis and S.Vourakis, Introducing HEP to schools through educational scenaria http://www.epjconferences.org/articles/epjconf/pdf/2015/14/epjconf_icnfp2014_03021.pdf 24. C. Kourkoumelis, "How "Discover the COSMOS", "PATHWAY", "Go-Lab" and "Inspiring Science Education" are changing the science education in European high schools", EPJ Web of Conferences 71, (2014) 00071

http://www.epjconferences.org/articles/epjconf/pdf/2014/08/epjconf_icnfp2013_00071.pdf 25. Stylianos Vourakis, Bringing high energy physics to the classroom with HY.P.A.T.I.A., ICNFP Kolympari 2013, EPJ Web of Conferences 71, (2014) 00137

26. Christine Kourkoumelis and Stylianos Vourakis on behalf of the ATLAS collaboration, How the HYPATIA analysis tool is used as a hands-on experience to introduce HEP to high schools, Presented at the ICHEP 2014,Valencia, Spain, Valencia 2014, Nuclear Physics B Proceedings Supplement 00 (2014) 1-7 : ICHEP 2014,Valencia, Spain, Valencia 2014, ... Supplement 00 (2014) 1-7 : 10.1016/j.nuclphysbps.2015.09.198

27. [Online] http://atlas-live-virtual-visit.web.cern.ch/atlas-live-virtual-visit/

28. [Online] http://cms.web.cern.ch/content/virtual-visits

29. [Online] http://www.hep.ucl.ac.uk/atlas/atlantis/

30. [Online] http://hypatia.phys.uoa.gr/

31. [Online] http://hypatia.iasa.gr/

32. Dimitris Fassouliotis, Christine Kourkoumelis, Stylianos Vourakis, The Inspiring Science Education project and the resources for HEP analysis by university students, 4th ICNFP Kolympari 2015,EPJ Web of Conferences 126, 02017 (2016),http://dx.doi.org/10.1051/epjconf/201612602017

33. [Online] http://opendata.atlas.cern/

34. [Online] http://hypatia.iasa.gr/en/Exercise_6_eng.pdf

35. [Online] http://hypatia.iasa.gr/en/batch.html

36. [Online] http://opendata.atlas.cern/externals/dev_index.php 